\title{
Comparison of long and short axis quantification of left ventricular volume parameters by cardiovascular magnetic resonance, with ex-vivo validation
}

\author{
Helene Childs ${ }^{1 \dagger}$, Lucia Ma ${ }^{1 \dagger}$, Michael Ma ${ }^{1}$, James Clarke ${ }^{2}$, Myra Cocker ${ }^{1}$, Jordin Green ${ }^{1,3}$, Oliver Strohm ${ }^{1}$ and \\ Matthias G Friedrich ${ }^{1 *}$
}

\begin{abstract}
Background: The purpose of the study was to compare the accuracy and evaluation time of quantifying left ventricular (LV), left atrial (LA) volume and LV mass using short axis (SAX) and long axis (LAX) methods when using cardiovascular magnetic resonance (CMR).

Materials and methods: We studied 12 explanted canine hearts and 46 patients referred for CMR (29 male, age $47 \pm 18$ years) in a clinical 1.5 T CMR system, using standard cine sequences. In standard short axis stacks of various slice thickness values in dogs and $8 \mathrm{~mm}$ slice thickness (gap $2 \mathrm{~mm}$ ) in patients, we measured LV volumes using reference slices in a perpendicular, long axis orientation using certified software. Volumes and mass were also measured in six radial long axis (LAX) views.

LV parameters were also assessed for intra- and inter-observer variability. In 24 patients, we also analyzed reproducibility and evaluation time of two very experienced ( $>10$ years of CMR reading) readers for SAX and LAX.

Results: In the explanted dog hearts, there was excellent agreement between ex vivo data and LV mass and volume data as measured by all methods for both, LAX $\left(r^{2}=0.98\right)$ and SAX $\left(r^{2}=0.88\right.$ to 0.98$)$. LA volumes, however, were underestimated by $13 \%$ using the LAX views. In patients, there was a good correlation between all three assessed methods ( $r^{2} \geq 0.95$ for all). In experienced clinical readers, left-ventricular volumes and ejection fraction as measured in LAX views showed a better inter-observer reproducibility and a 27\% shorter evaluation time.

Conclusion: When compared to an ex vivo standard, both, short axis and long axis techniques are highly accurate for the quantification of left ventricular volumes and mass. In clinical settings, however, the long axis approach may be more reproducible and more time-efficient. Therefore, the rotational long axis approach is a viable alternative for the clinical assessment of cardiac volumes, function and mass.
\end{abstract}

\section{Background}

Cardiovascular magnetic resonance imaging (CMR) is well recognized as a gold standard for functional imaging and assessment. CMR using state-of-the-art sequences has been shown to be very reproducible, is considered appropriate for many clinical indications [1] and there is consensus about its clinical use and methodology [2]; newer sequences, however, have not been evaluated

\footnotetext{
* Correspondence: matthias.friedrich@ucalgary.ca

+ Contributed equally

${ }^{1}$ Stephenson Cardiovascular MR Centre at the Libin Cardiovascular Institute of Alberta, Departments of Cardiac Sciences and Radiology, University of

Calgary, AB, Canada

Full list of author information is available at the end of the article
}

against an ex vivo gold standard. Furthermore, short axis (SAX) stacks, considered the standard approach for quantifying left ventricular (LV) volumes and function and used as such for validation [3], are time consuming and there are partial volume effects leading to problems that arise upon defining the slice area in the most basal and most apical slices [4]. Inclusion or exclusion of basal slices is of particular interest since until now, the blood volume in the LV outflow tract is often excluded, while atrial volume may be erroneously included. Likely due to this problem, the utilization of SAX views may be hampered by a lesser reproducibility [5].

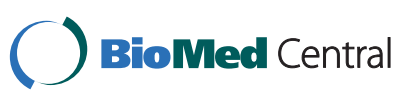

() 2011 Childs et al; licensee BioMed Central Ltd. This is an Open Access article distributed under the terms of the Creative Commons Attribution License (http://creativecommons.org/licenses/by/2.0), which permits unrestricted use, distribution, and reproduction in any medium, provided the original work is properly cited. 
Long axis views (LAX) have been proposed as an alternative to SAX-derived methods [6]. We aimed to validate state-of-the-art sequences in SAX and rotational LAX views in explanted canine hearts and to compare the two methods in clinical CMR studies. This is of clinical importance for LV function and mass analysis, but also for correlating these results with tissue abnormalities such as high signal intensity areas in late Gd enhancement or T2-weighted images.

\section{Materials and methods}

All examinations were performed on a $1.5 \mathrm{~T}$ system (MAGNETOM Avanto ${ }^{\circledR}$, Siemens Healthcare, Erlangen, Germany). All quantitative CMR parameters were assessed using certified software $\left(\mathrm{cmr}^{42}\right.$, Circle Cardiovascular Imaging Inc., Calgary, Canada). All readers had training on drawing contours with special emphasis on how to avoid misinterpretation of the basal slice.

\section{Ex vivo studies}

Twelve freshly explanted canine hearts were filled with MRI-compatible, autopolymerizing acrylic resin (Dentsply, Caulk, York, PA, USA) for an accurate representation of cavities, and then imaged with both radial LAX and SAX orientations for comparison. Three contiguous SAX stacks with full LA and LV coverage were analyzed separately (slice thickness: $10 \mathrm{~mm}, 8 \mathrm{~mm}$, and $5 \mathrm{~mm}$ ) and six radial LAX slices were obtained as per published protocols [5]. CMR dimensions were assessed (Figure 1) in a blinded fashion and results were compared to mold data. Molds were excised and volumes were determined by, displacement, a digital scale was used to weigh LV myocardium.

\section{Subjects}

We assessed 46 individuals, (29 male, 17 female; mean age $47 \pm 18$, range $15-79$ years). 4 were healthy volunteers without evidence for heart disease, while 42 patients were randomly selected from clinical referrals for functional imaging, tissue characterisation and/or viability. The study was approved by the local ethics committee and informed consent was obtained from each individual.

\section{CMR sequence}

Standard Steady-State-Free-Precession (SSFP) cine sequences were used for all approaches. Typical parameters were: TE $1.1 \mathrm{~ms}$, TR $2.31 \mathrm{~ms}$, FA $75^{\circ}$, matrix $340 \times$ $284 \mathrm{~mm}$, pixel size $1.3 \mathrm{~mm} \times 1.4 \mathrm{~mm}$ and an IPAT factor 2 . With 25 lines per segment, the effective repetition time typically was $57 \mathrm{~ms}$. SAX acquisition was performed as multiple short axes across the entire LV in an imaging plane perpendicular to the LAX of the LV with a slice thickness of $8 \mathrm{~mm}$ and a $2 \mathrm{~mm}$ gap. The LAX slices were performed using 6 slices rotating in 30 degree increments around the anatomical LV LAX.

\section{Image Analysis}

CMR image analysis was performed using certified software $\left(\mathrm{cmr}^{42}\right.$, Circle Cardiovascular Imaging Inc., Calgary,

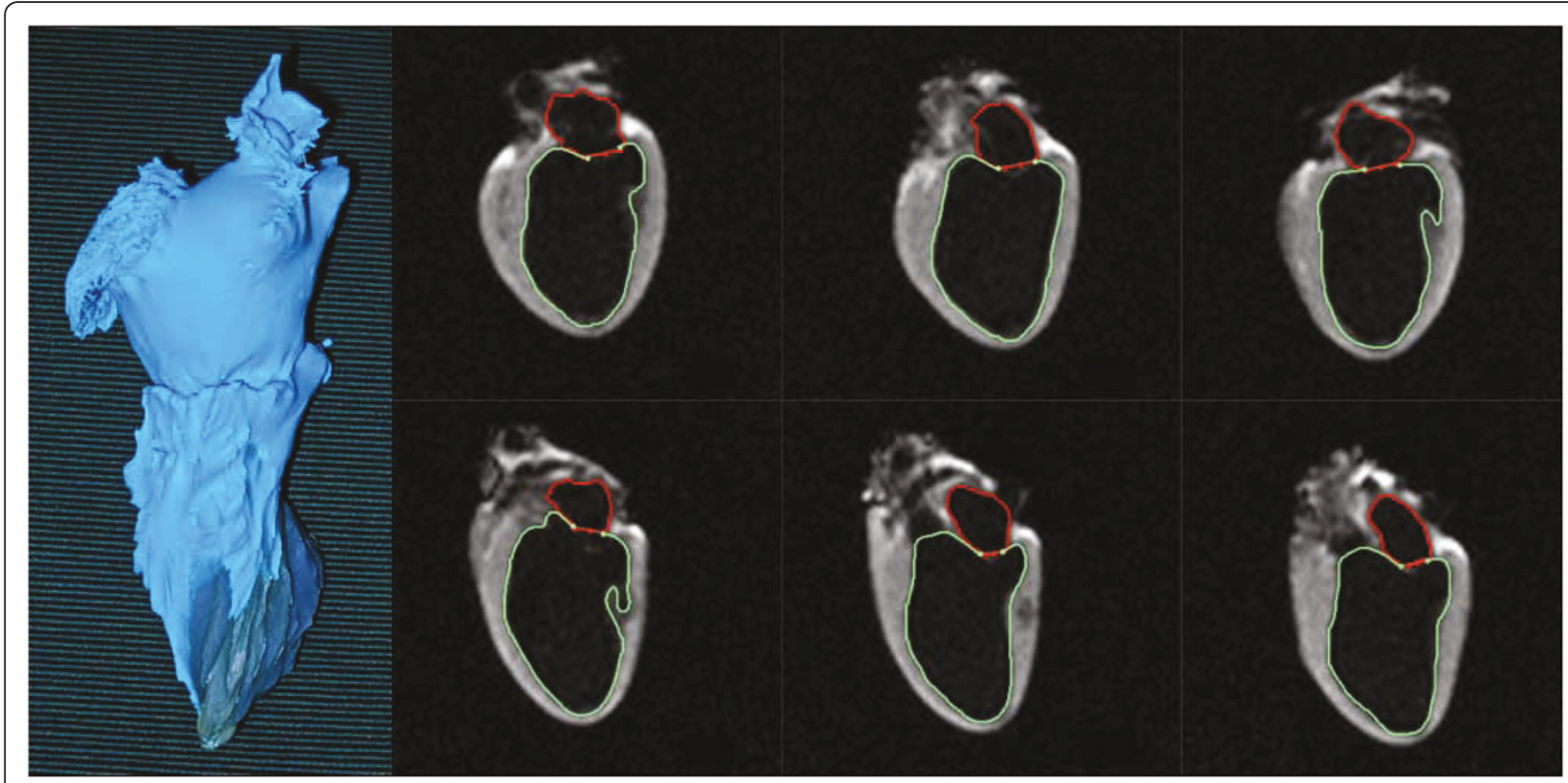

Figure 1 Mold of explanted dog heart and long axis CMR images. Left: Mold of the left ventricle and left atrium of an explanted dog heart. Right: Six ex vivo CMR long axis views of the mold-filled heart. Contours are shown for the subendocardial border of the left atrium and left ventricle. 
Canada) by well-trained observers. Endocardial and epicardial contours were drawn manually for the LV at endsystole and end-diastole in each data set.

In contrast to an uncontrolled approach for identifying the most basal short axis slice by identifying the most basal image which contains at least $50 \%$ of circumferential myocardium [7], we used perpendicular images with lines representing the position of the basal and the apical short axis planes as automatically provided by the software to control for the position and decide on the inclusion of this slice (Figure 2). The blood volume encompassed by the mitral valve was excluded while LV outflow tract volume was included as determined by the LAX cross-reference. Papillary muscles and trabeculations were included in LV mass and volume calculations. The interventricular septum was included in the LV mass. As LAX cross-reference views, 3- and 4-chamber views were typically used (Figure 3).

As for SAX views, endocardial and epicardial contours were manually drawn in end-systolic and end-diastolic frames of all LAX views. Contours excluded trabeculations while including most of the papillary muscles. The blood volume in the mitral valve was excluded with inclusion of blood volume in the LV outflow tract up to the aortic valve (Figure 4).

We assessed intra-observer variability by having one observer analyzing volumes with SAX twice, while a different observer analyzed volumes with LAX approach twice. To minimize observer bias, each of the two less experienced readers read only one of the orientations, either SAX or LAX. This may have introduced a bias for the time needed. Both readers, however, had similar training before the study. Furthermore, for experienced readers such a bias can be excluded, since they read both, SAX and LAX view in separate sessions.

For all methods, end-diastolic volume (EDV), end-systolic (ESV), stroke volume (SV), ejection fraction (EF), and LV mass were assessed by three independent observers. LV mass was measured at end-systole by both methods because of the easier definition of the endocardial borders in the contracted myocardium. The time needed to complete assessments was recorded in a randomly selected subgroup of 12 individuals.

\section{Statistical Analysis}

CMR data was compared to mold measurements using a paired t test and Pearson's correlation coefficient. Inter-

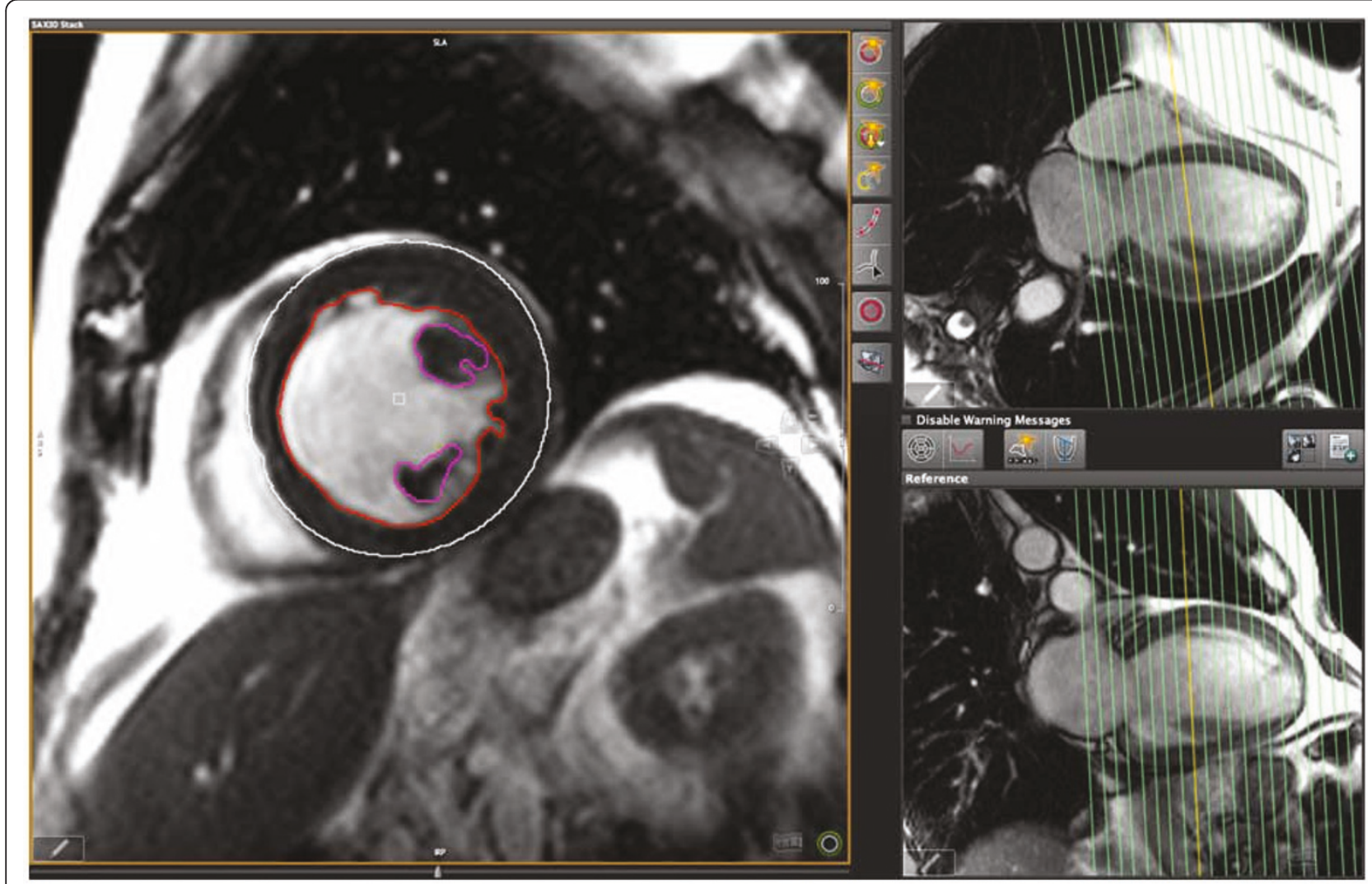

Figure 2 LV function analysis in short axis images using long axis views as a cross-reference. Left panel: Diastolic mid-ventricular short axis view with contours. Right upper panel: Example long axis reference view in a 4-chamber orientation. Right lower panel: Example long axis reference view in a 2-chamber orientation. The orange lines represent the location of the cross-sectional long axis views used for measurements. 


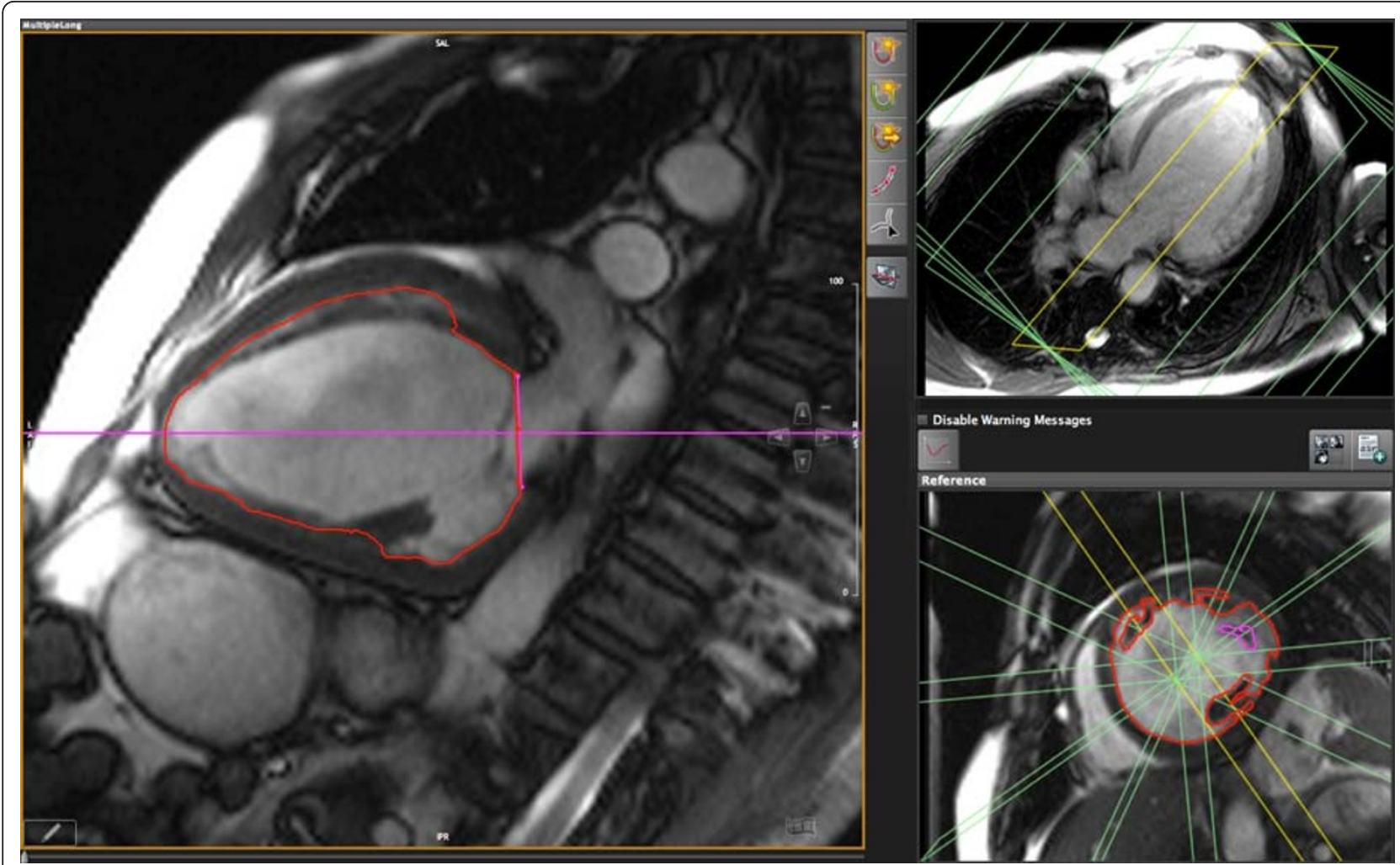

Figure 3 LV function analysis in long axis images using short axis views as a cross-reference. Left panel: Diastolic long axis view with endocardial contour. Right upper panel: Example short axis reference view in a 3-chamber orientation. Right lower panel: Example short axis reference view in short axis orientation. The orange lines represent the location of the cross-sectional views used for measurements.

and intra-observer variability was analyzed using linear regression to calculate the square of the correlation coefficient, $\left(\mathrm{r}^{2}\right)$. The mean difference between observations was calculated by averaging the absolute value of the difference between methods. Likewise, the difference for inter- and intra-observer variability was calculated as the absolute value of the difference divided by the initial measurement. Both absolute and relative differences between methods were calculated and Bland-Altman plots calculated (Figure 5, 6 and 7). All calculations were completed using statistics software (Microsoft Excel 12.2.6 for Mac, Microsoft Corporation, USA).

\section{Results}

\section{Explanted canine hearts}

LV results obtained from SAX and LAX views were in excellent agreement with the results from the molds (Pearson's correlation coefficient, $\mathrm{r} \geq 0.6, \mathrm{p}<0.05$ ). LA volumes were significantly correlated with SAX methods $(\mathrm{r} \geq 0.6, \mathrm{p}<0.05)$ (table 1). There was no significant difference between LAX and SAX techniques for measurements of LV volumes, LA volumes or LV mass ( $\mathrm{p}>$ $0.05)$. There was also no significant difference between
CMR-derived measurements and actual values of LV mass (explanted hearts) and LV volumes (mold).

\section{Patients}

Seventeen of the 42 patients referred for CMR did not reveal any abnormalities, while 25 had various cardiac conditions. Image quality was good in all cases, none of the patients were excluded from the analysis.

There was a good correlation with no significant difference between SAX and LAX evaluation (Table 2).

Inter-observer variability was smaller with LAX measurements for LVEDV, LVESV and LV-EF (Table 3). Results of LV mass quantification did not differ between observers ( $\mathrm{p}=\mathrm{NS})$.

For less experienced readers, the time of evaluation for SAX was 13 minutes and 8 seconds, while the time of evaluation for LAX was slightly less, 12 minutes and 42 seconds. Required evaluation times for experienced readers are shown in table 4 .

\section{Discussion}

Our data indicate that both, cross-referenced short axis and long axis views provide accurate measurements of 


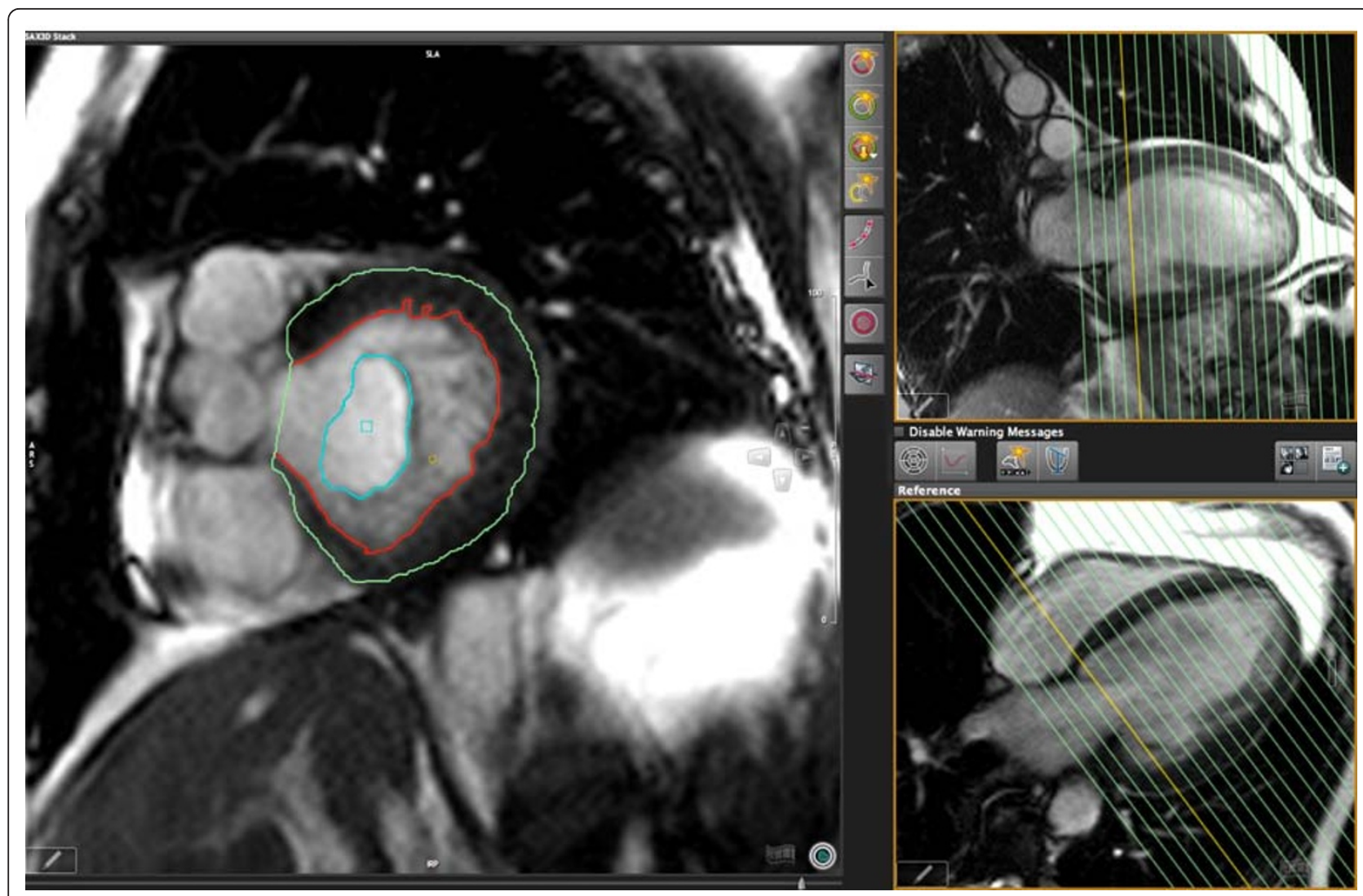

Figure 4 Correction of atrial volume next to the mitral valve. Left panel: Diastolic basal short axis view with contours. The green contour encircles the atrial portion of the volume in this slice and is excluded from the ventricular volume. Right upper panel: Example long axis reference view in a 2-chamber orientation. Right lower panel: Example long axis reference view in a 4-chamber orientation. The orange lines represent the location of the cross-sectional long axis views used for measurements.

volumetric LV parameters. This is not specific for CMR and has implications for other imaging techniques such as CT. Furthermore, our data indicate that long axis views take less time for evaluation. Another advantage of long axis views is the low susceptibility to errors induced by misinterpretation of slices close to the mitral valve. In fact, a post-hoc analysis of our data showed that errors of standard short axis data were due to inclusion or exclusion of the most basal slice, while there was no difference between cross-referenced and long axis results. Therefore, long axis planes may be a useful approach in most routine clinical situations.

LV mass was larger when calculated by SAX. This could be due to the inclusion of trabeculations and papillary muscles in SAX while only papillary muscles were included in LAX; however, we were concerned that including both trabeculations and papillary muscles in the LAX would lead to an over-estimation in LV mass due to the use of rotational volumetry calculation [5]. Another possible explanation is that SAX images experience partial volume effects in basal and apical slices while in radial LAX images the identification of subendocardial borders is easier.

Most validation studies have been performed using SAX measurements of LV mass [8-10]. Furthermore, tissue characterization often is performed in SAX views and therefore this view may be better for correlating results between sequences. Since abnormalities seen in images used for tissue characterization should be verified by cross-sectional views and therefore both long and short axis views are required anyway, a comparison of tissue characteristics with the cine images is equally possible. More recent studies indicate that long-axis based methods may be equally accurate yet may have a smaller intraobserver variability [6]. This may be due to the clear visualisation of both mitral and aortic valve planes and the reduction of partial volume effects allowing for welldefined myocardial boundaries [5]. So, while LAX methods use geometric assumptions, the advantage of having a perpendicular view on the basal and apical borders of the left ventricle may outweigh the disadvantage of partially replacing actual measurements by computed data. 

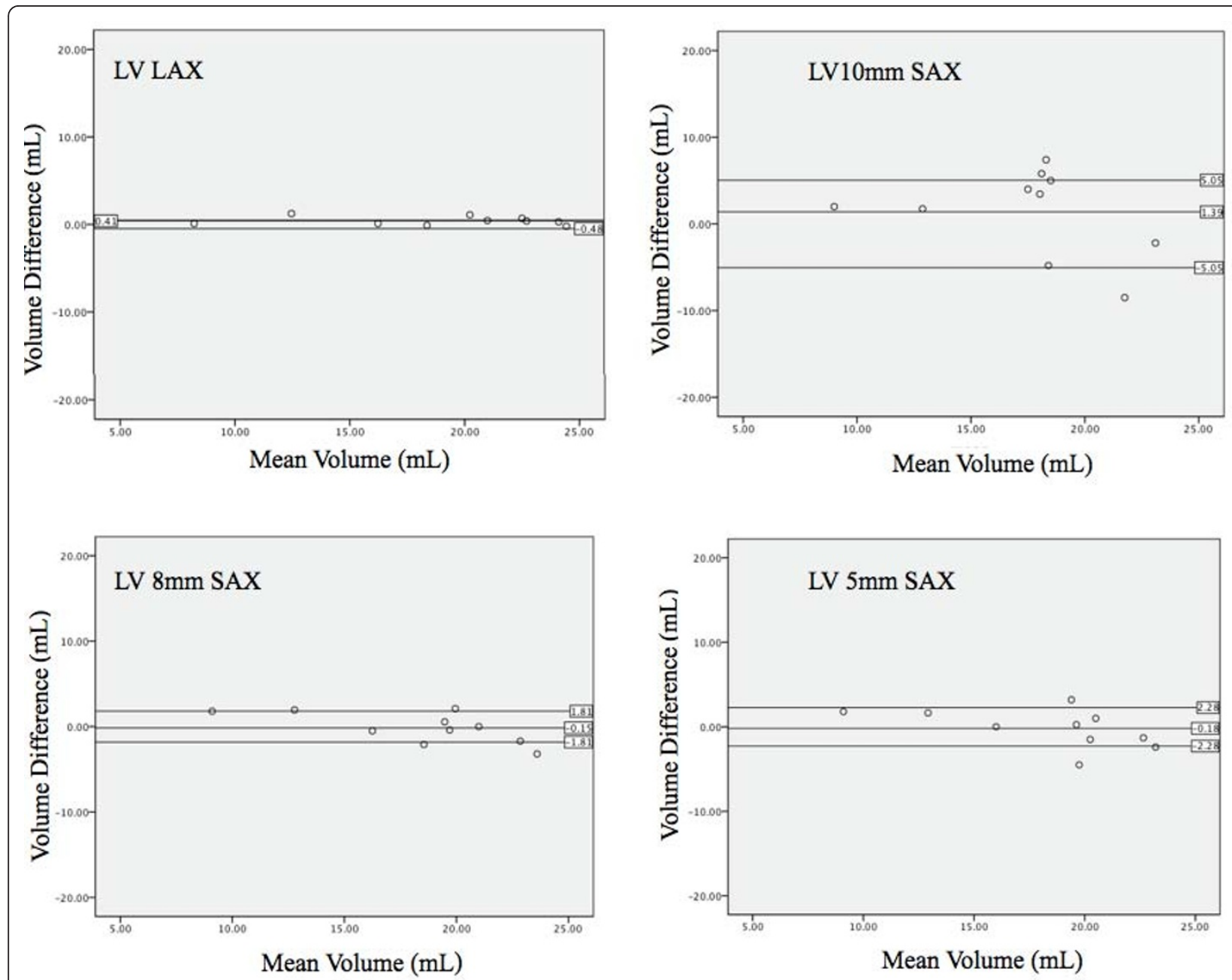

Figure 5 Bland-Altman analysis of left ventricular volume. Overall bias and 95\% limits of agreement between mold data and different methods for LV volume determination. Standard compared to CMR measurements using radial (top left), short axis $10 \mathrm{~mm}$ (top right), short axis $8 \mathrm{~mm}$ (bottom left), and short axis $5 \mathrm{~mm}$ (bottom right) techniques. The long axis method showed the least amount of variation and was on average closest to the real volume.

\section{Limitations}

We did not systematically assess the impact of ventricular shape criteria on our results. Although this has not been studied systematically, a highly irregular morphology may limit the accuracy of LAX data [11]. In the LAX views, traceless and papillary muscles were only partially included into LV mass, whereas they were completely included in the SAX stack evaluation. This is due to the fact, that inclusion of small structures (i.e. smaller than the distance tracked by the $30^{\circ}$ rotation between LAX planes) leads to overestimation of the volume of these tissues (unpublished data). This inconsistency between the methods however was not associated with significant differences between methods; yet, it may explain the non-significant trend toward underestimation of LV mass by LAX. For clinical scenarios, the lack of significant differences let this problem appear irrelevant. In the SAX method, we used no gaps for the dog studies, but had gaps in patients. The reason for using no gaps in dogs was simply to compare the results by both methods excluding other confounders. Resembling clinical scenarios, however, we applied gaps in patient studies as typically done in clinical applications and clinical research [12]. The applicability of our results to other readers may be limited by individual approaches how to deal with inclusion or exclusion of papillary muscles and trabecular tissue. 

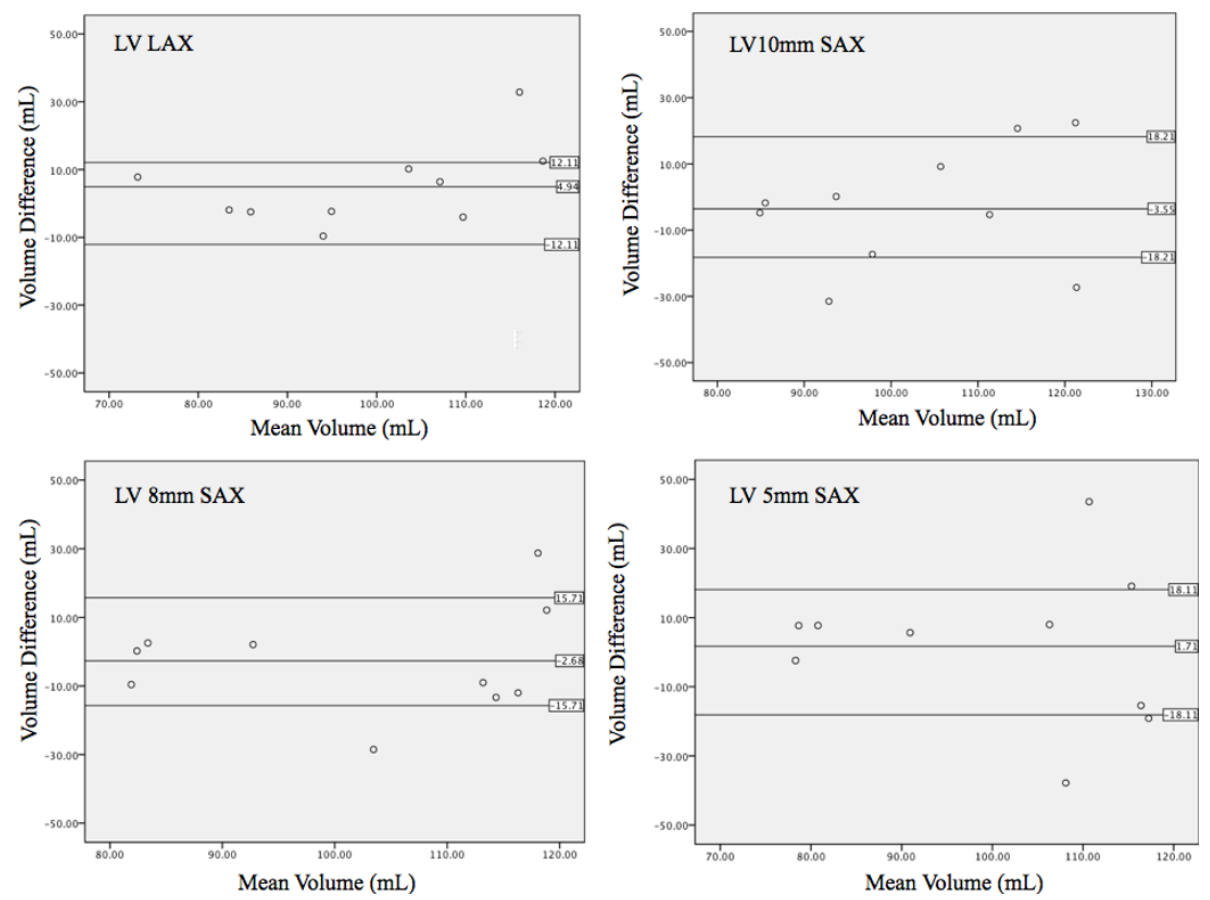

Figure 6 Bland-Altman analysis of left ventricular mass. Overall bias and 95\% limits of agreement between mold data and different methods for LV volume determination. Standard compared to CMR measurements using radial (top left), short axis $10 \mathrm{~mm}$ (top right), short axis $8 \mathrm{~mm}$ (bottom left), and short axis $5 \mathrm{~mm}$ (bottom right) techniques.
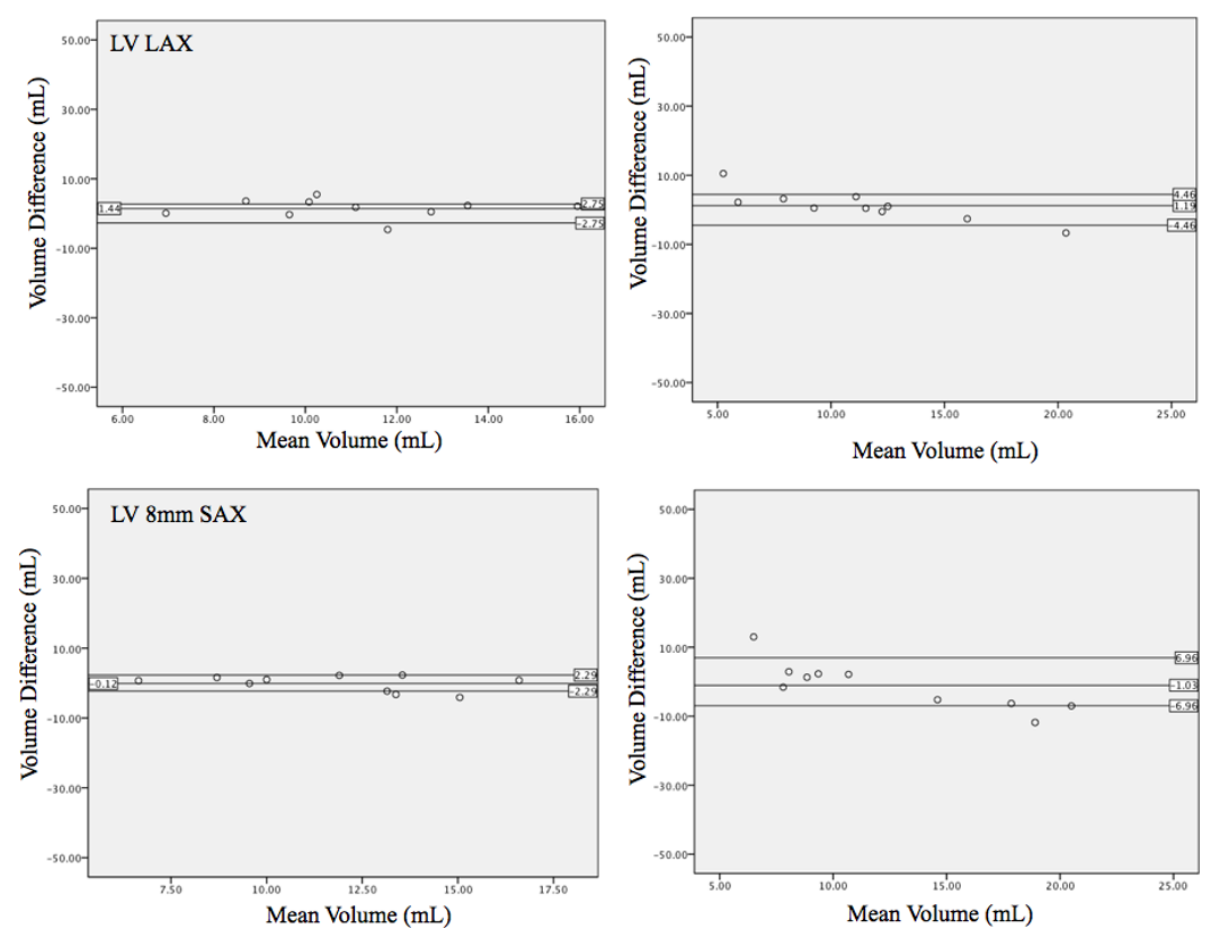

Figure 7 Bland-Altman analysis of left atrial volume. Overall bias and 95\% limits of agreement between mold data and different methods for LV volume determination. Standard compared to CMR measurements using radial (top left), short axis $10 \mathrm{~mm}$ (top right), short axis $8 \mathrm{~mm}$ (bottom left), and short axis $5 \mathrm{~mm}$ (bottom right) techniques. 
Table 1 Differences between CMR measurements and actual results with correlation coefficients in explanted canine hearts

\begin{tabular}{|c|c|c|c|c|c|}
\hline & Reference (mold) & LAX & SAX $10 \mathrm{~mm}$ & SAX $8 \mathrm{~mm}$ & SAX $5 \mathrm{~mm}$ \\
\hline LV Volume & $23.3 \pm 5.3 \mathrm{ml}$ & $\begin{aligned}-2.5 & \pm 1.1 \% \\
C V & =1.18\end{aligned}$ & $\begin{aligned}-7.0 & \pm 7.8 \% \\
C V & =3.63\end{aligned}$ & $\begin{array}{l}-0.7 \pm 4.6 \% \\
C V=-12.1\end{array}$ & $\begin{array}{c}+0.3 \pm 5.3 \% \\
C V=-12.7\end{array}$ \\
\hline LA Volume & $11.8 \pm 7.5 \mathrm{ml}$ & $\begin{array}{c}-13.3 \pm 7.0 \% \\
C V=1.61\end{array}$ & $\begin{aligned}-4.2 & \pm 6.9 \% \\
C V & =3.49\end{aligned}$ & $\begin{array}{c}-3.3 \pm 6.9 \% \\
C V=6.07\end{array}$ & $\begin{array}{c}+5.3 \pm 9.1 \% \\
C V=-9.24\end{array}$ \\
\hline LV Mass & $99.6 \pm 5.5 \mathrm{~g}$ & $\begin{aligned}-3.3 & \pm 3.0 \% \\
C V & =2.68\end{aligned}$ & $\begin{array}{c}+7.5 \pm 5.6 \% \\
C V=-3.49\end{array}$ & $\begin{array}{c}+4.0 \pm 4.2 \% \\
C V=-5.31\end{array}$ & $\begin{array}{r}+0.1 \pm 6.0 \% \\
\mathrm{CV}=17.3\end{array}$ \\
\hline
\end{tabular}

Difference CV shows the degree of variation by providing the ratio of the standard deviation relative to the mean. Values are expressed as mean \pm SE. LV: Left ventricular; LA: Left atrial; LAX: Long axis method; SAX: Short axis method; CV: coefficient of variation of the difference between mold and CMR values.

Table 2 Volumetric results as calculated from the different analysis methods in patients

\begin{tabular}{ccc}
\hline & Short axis views & Long axis views \\
\hline LVEDV & $177 \pm 99 \mathrm{ml}$ & $182 \pm 101 \mathrm{ml}$ \\
\hline LVESV & $94 \pm 102 \mathrm{ml}$ & $91 \pm 97 \mathrm{ml}$ \\
\hline LV-EF & $55 \pm 16 \%$ & $55 \pm 15 \%$ \\
\hline LV mass & $144 \pm 64 \mathrm{~g}$ & $141 \pm 62 \mathrm{~g}$ \\
\hline
\end{tabular}

None of the differences was statistically significant.

Table 3 Absolute inter-observer differences (\%) using different acquisition orientations in patients

\begin{tabular}{rcc}
\hline & Short axis views & Long axis views \\
\hline LVEDV & $11.2 \pm 7.9 \mathrm{ml}$ & $4.4 \pm 4.6 \mathrm{ml}^{*}$ \\
\hline LVESV & $18.6 \pm 12.5 \mathrm{ml}$ & $7.0 \pm 5.1 \mathrm{ml}^{*}$ \\
\hline LV-EF & $7.2 \pm 5.7 \%$ & $3.0 \pm 1.7 \% \%^{*}$ \\
\hline LV mass & $14.0 \pm 14.4 \mathrm{~g}$ & $7.1 \pm 6.8 \mathrm{~g}$
\end{tabular}

*: $p<0.05$ vs. standard and cross-referenced short axis views. Differences between standard and cross-referenced short axis view measurements were not significant.

Table 4 Time needed for evaluation using different acquisition orientations in patients

\begin{tabular}{rccc}
\hline & $\begin{array}{c}\text { Short axis } \\
\text { views }\end{array}$ & $\begin{array}{c}\text { Long axis } \\
\text { views }\end{array}$ & $\begin{array}{c}\text { Time } \\
\text { saving }\end{array}$ \\
\hline Experienced reader & $8: 42 \pm 4: 38$ & $6: 24 \pm 0: 49^{*}$ & $26 \%$ \\
\hline $\begin{array}{r}\text { Less experienced } \\
\text { reader }\end{array}$ & $13: 08 \pm 7: 14$ & $12: 42 \pm 3: 34$ & $3 \%$
\end{tabular}

*: $\mathrm{p}<0.05$ vs. standard and cross-referenced short axis views.

\section{Conclusion}

When compared to an ex vivo standard, both, short axis and long axis techniques are highly accurate for the quantification of left ventricular volumes and mass. In patients, however, the long axis approach may be more reproducible and more time-efficient. For the analysis of left ventricular volumes by tomographic techniques, mass and function in hearts without severe shape alterations, a long axis approach may be a viable alternative

\section{Acknowledgements}

We are thankful for the wonderful assistance provided by Loreen Thon and Sheri-Lee Rinella. We also want to thank the laboratory of Dr. John Tyberg for providing the hearts.

\section{Author details}

${ }^{1}$ Stephenson Cardiovascular MR Centre at the Libin Cardiovascular Institute of Alberta, Departments of Cardiac Sciences and Radiology, University of Calgary, AB, Canada. ${ }^{2}$ Dept. of Nuclear Medicine and Radiology, Dalhousie University, Halifax, NC, Canada. ${ }^{3}$ Siemens Healthcare, Henkestr. 12791054 Erlangen, Germany.

\section{Authors' contributions}

LM, MM, MC, and JC were involved with the patient component of the study and assisted in drafting the manuscript. HC completed the ex-vivo component of the study and assisted in drafting the manuscript. JG was involved in both patient and ex-vivo scanning components.

MF and OS designed and coordinated this study. All authors read and approved the final manuscript.

\section{Competing interests}

Oliver Strohm is an advisor to Circle CV Imaging Inc., Calgary, Canada. and Matthias G. Friedrich is on the board of directors of Circle CV Imaging Inc., Calgary, Canada.

Received: 12 April 2011 Accepted: 11 August 2011

Published: 11 August 2011

\section{References}

1. Hendel RC, Patel MR, Kramer CM, Poon M, Hendel RC, Carr JC, Gerstad NA, Gillam LD, Hodgson JM, Kim RJ, Kramer CM, Lesser JR, Martin ET, Messer JV, Redberg RF, Rubin GD, Rumsfeld JS, Taylor AJ, Weigold WG, Woodard PK, Brindis RG, Hendel RC, Douglas PS, Peterson ED, Wolk MJ, Allen JM, Patel MR: ACCF/ACR/SCCT/SCMR/ASNC/ NASCI/SCAI/SIR 2006 appropriateness criteria for cardiac computed tomography and cardiac magnetic resonance imaging: a report of the American College of Cardiology Foundation Quality Strategic Directions Committee Appropriateness Criteria Working Group, American College of Radiology, Society of Cardiovascular Computed Tomography, Society for Cardiovascular Magnetic Resonance, American Society of Nuclear Cardiology, North American Society for Cardiac Imaging, Society for Cardiovascular Angiography and Interventions, and Society of Interventional Radiology. J Am Coll Cardiol 2006, 48:1475-97.

2. Hundley WG, Bluemke DA, Finn JP, Flamm SD, Fogel MA, Friedrich MG, Ho VB, Jerosch-Herold M, Kramer CM, Manning WJ, Patel M, Pohost GM, Stillman AE, White RD, Woodard PK: ACCF/ACR/AHA/NASCI/SCMR 2010 Expert Consensus Document on Cardiovascular Magnetic Resonance: A Report of the American College of Cardiology Foundation Task Force on Expert Consensus Documents. Circulation 2010, 121:2462-508.

3. François CJ, Fieno DS, Shors SM, Finn JP: Left ventricular mass: manual and automatic segmentation of true FISP and FLASH cine MR images in dogs and pigs. Radiology 2004, 230:389-95.

4. Dulce MC, Mostbeck GH, Friese KK, Caputo GR, Higgins CB: Quantification of the left ventricular volumes and function with cine MR imaging: 
comparison of geometric models with three-dimensional data. Radiology

1993, 188:371-6.

5. Bloomer TN, Plein S, Radjenovic A, Higgins DM, Jones TR, Ridgway JP: Sivananthan MU Cine MRI using steady state free precession in the radial long axis orientation is a fast accurate method for obtaining volumetric data of the left ventricle. J Magn Reson Imaging 2001, 14:685-92.

6. Bloomgarden DC, Fayad ZA, Ferrari VA, Chin B, Sutton MG, Axel L: Global cardiac function using fast breath-hold MRI: validation of new acquisition and analysis techniques. Magn Reson Med 1997, 37:683-92.

7. Papavassiliu T, Kühl HP, Schröder M, Süselbeck T, Bondarenko O, Böhm CK, Beek A, Hofman MM, van Rossum AC: Effect of endocardial trabeculae on left ventricular measurements and measurement reproducibility at cardiovascular MR imaging. Radiology 2005, 236:57-64

8. Keller AM, Peshock RM, Malloy CR, Buja LM, Nunnally R, Parkey RW, Willerson JT: In vivo measurement of myocardial mass using nuclear magnetic resonance imaging. J Am Coll Cardiol 1986, 8:113-7.

9. Shapiro MD, Nieman K, Nasir K, Nomura CH, Sarwar A, Ferencik M, Abbara S, Hoffmann U, Gold HK, Jang IK, Brady TJ, Cury RC: Utility of Cardiovascular Magnetic Resonance to Predict Left Ventricular Recovery After Primary Percutaneous Coronary Intervention for Patients Presenting With Acute ST-Segment Elevation Myocardial Infarction. The American Journal of Cardiology 2007, 100:211-6.

10. Katz J, Milliken MC, Stray-Gundersen J, Buja LM, Parkey RW, Mitchell JH, Peshock RM: Estimation of human myocardial mass with MR imaging Radiology 1988, 169:495-8.

11. Friedrich MG, Schulz-Menger J, Strohm O, Dick AJ, Dietz R: The diagnostic impact of $2 \mathrm{D}$ - versus $3 \mathrm{D}$ left ventricular volumetry by MRI in patients with suspected heart failure. Magma 2000, 11:16-9.

12. Hudsmith LE, Petersen SE, Francis JM, Robson MD, Neubauer S: Normal human left and right ventricular and left atrial dimensions using steady state free precession magnetic resonance imaging. J Cardiovasc Magn Reson 2005, 7:775-82.

doi:10.1186/1532-429X-13-40

Cite this article as: Childs et al: Comparison of long and short axis quantification of left ventricular volume parameters by cardiovascular magnetic resonance, with ex-vivo validation. Journal of Cardiovascular Magnetic Resonance 2011 13:40.

\section{Submit your next manuscript to BioMed Central and take full advantage of:}

- Convenient online submission

- Thorough peer review

- No space constraints or color figure charges

- Immediate publication on acceptance

- Inclusion in PubMed, CAS, Scopus and Google Scholar

- Research which is freely available for redistribution

Submit your manuscript at www.biomedcentral.com/submit 